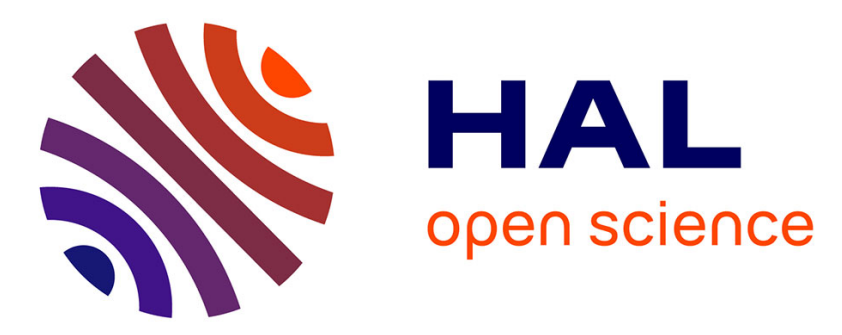

\title{
Idéal linguistique, pratiques langagières et construction des savoirs \\ Catherine Taine-Cheikh
}

\section{To cite this version:}

Catherine Taine-Cheikh. Idéal linguistique, pratiques langagières et construction des savoirs. Abdel Wedoud Ould Cheikh. Etat et société en Mauritanie. Cinquante ans après l'Indépendance, IFM Karthala, pp.261-285, 2014. halshs-01098727

\section{HAL Id: halshs-01098727 https://shs.hal.science/halshs-01098727}

Submitted on 28 Dec 2014

HAL is a multi-disciplinary open access archive for the deposit and dissemination of scientific research documents, whether they are published or not. The documents may come from teaching and research institutions in France or abroad, or from public or private research centers.
L'archive ouverte pluridisciplinaire HAL, est destinée au dépôt et à la diffusion de documents scientifiques de niveau recherche, publiés ou non, émanant des établissements d'enseignement et de recherche français ou étrangers, des laboratoires publics ou privés. 


\title{
Idéal linguistique, pratiques langagières et construction des savoirs
}

\author{
Catherine TAINE-CHEIKH \\ LACITO (CNRS - Villejuif)
}

\section{Introduction}

Je me souviens de la première fois où j'ai discuté avec un des deux étudiants mauritaniens qui étaient inscrits dans la même classe d'hypokhâgne que moi ${ }^{1}$. C'était à l'automne 69, dans un petit café de la rue Saint-André des Arts situé tout près du lycée Fénelon, en plein Quartier latin. J'étais alors bien loin de me douter que Marie-Thérèse Daddah, l'épouse du Président de la République Islamique de Mauritanie, avait fait ellemême une partie de ses études dans ce même lycée (et je doute que mon interlocuteur en ait eu lui-même alors connaissance). J'ignorais également que la Mauritanie jouerait un aussi grand rôle dans ma vie - par le hasard des choses qui faisait qu'à l'époque le lycée Fénelon était l'un des rares établissements où le choix de l'arabe comme première langue vivante était possible.

L'arabe, déjà... Mais, à l'époque, je n'avais pas plus cette langue que ce pays dans mon champ de vision et, de cette discussion, j'ai surtout gardé le souvenir d'une curiosité, mêlée d'une gêne quelque peu honteuse, face à mon ignorance presque totale de ce lointain pays africain. Il est vrai qu'on savait alors très peu de choses, en France, de cette jeune République qui avait émergé dans la foulée du mouvement général de décolonisation. Les manuels scolaires ne consacraient pas un chapitre à cette période d'histoire toute récente et la guerre du Sahara n'avait pas encore attiré les feux de l'actualité sur cette région du monde.

La langue arabe, je n'ai commencé vraiment à m'y intéresser qu'à l'automne 71 , d'abord simplement pour apprendre à la parler, puis, quelques mois plus tard, pour en faire un objet d'étude et de recherche. En novembre 2010, pour les cinquante ans d'indépendance de la Mauritanie, je pouvais donc presque arguer, à mon niveau personnel, de quatre décennies de recherche. En terme de publications, cela signifie une petite douzaine de volumes édités et plus d'une centaine d'articles rédigés. Mais pour les Mauritaniens, quel intérêt ces travaux peuvent-ils avoir ? Je crains que, malheureusement pour moi (et peut-être aussi — oserai-je le prétendre — pour eux), mon domaine de recherche apparaisse souvent comme trop proche et trop lointain à la fois. Trop proche, parce qu'on se persuade facilement qu'une langue qu'on parle est une langue sur laquelle on n'a rien à apprendre. Trop lointain, parce que, les affaires linguistiques ne pouvant guère être traitées sans un brin de technicité, cela tend bien souvent à décourager les non initiés.

Proposer une sorte de synthèse en quelques pages relève donc, à plus d'un titre, du défi impossible. À défaut, je souhaite ici apporter quelques éléments de réflexion susceptibles de faire écho à mes recherches, tout en rendant hommage à quelques

\footnotetext{
${ }^{1}$ Ces deux étudiants en classe préparatoire à l'École Normale Supérieure de Saint Cloud étaient Sidaty Benhameida et Mohamed Haibetna ould Sidi Haiba.
} 
personnes qui ont joué un rôle notable dans les champs qui sont miens. J'espère montrer ce faisant que l'étude des langues, loin de servir seulement pour parler, ouvre aussi des portes sur la culture présente et passée, notamment en fournissant quelques clés pour déchiffrer l'histoire.

\section{L'idéal linguistique et le champ du politique}

Dans le monde, au cours des siècles, certaines langues en ont chassé d'autres, tandis qu'une majorité d'idiomes - sans nécessairement se délocaliser du lieu qui les a vues se former - présentent des marques plus ou moins importantes et radicales d'évolution interne, d'emprunts et de métissages.

En Afrique de l'Ouest, en particulier dans sa partie sahélo-saharienne, l'implantation du français est une greffe tardive, dont l'empreinte s'est approfondie au moment de l'indépendance et dans les quelques décennies qui l'ont précédée. Apporté dans les bagages des colonisateurs, le français s'impose tout d'abord comme l'une des langues officielles du nouvel État. C'est la langue de l'administration, celle dans laquelle ont été formés les premiers cadres de la jeune nation et dans laquelle furent enseignées les premières générations de bacheliers de l'enseignement moderne.

Très vite, cependant, les protestations contre la place prépondérance accordée à cette langue étrangère prennent de l'ampleur et les affrontements inter-ethniques de 1966 montrent qu'une partie au moins de la société mauritanienne renoue avec la résistance opposée à la langue du colonisateur durant la première moitié du $\mathrm{XX}^{\mathrm{e}}$ siècle. L'image du français, comme langue de la modernité et de l'unité nationale, est combattue au nom de la défense de l'arabe, cette langue devenant, en particulier dans la communauté des Bị̣̂̂an, l'étendard de revendications nationalistes et nationalitaires.

Je ne referai pas ici l'historique de la politique linguistique et de ses lourdes conséquences pour les relations entre les différentes communautés mauritaniennes. L'évolution du rapport de force entre le français et l'arabe se laisse lire dans les programmes scolaires des différentes filières de l'enseignement, dans le poids attribué aux examens à l'une et l'autre langue, dans les nominations aux postes de direction. Pour avoir connu une époque où la répartition des postes de directeur et directeur adjoint d'une administration se faisait encore en tenant compte d'un équilibre à respecter entre arabisant (voire bilingue) et francophone, il m'est facile de comprendre combien décisive pouvait être, pour l'avenir de beaucoup de Mauritaniens, le choix national de la langue privilégiée d'enseignement et de travail.

Pour les Halpulaaren, les Soninké et les Wolofs qui aspiraient à trouver un travail en dehors du secteur informel, le français ne pouvait qu'apparaître comme la meilleure planche de salut et, si quelques-uns se sont laissé convaincre par les mirages d'un enseignement en langue nationale, la plupart préféraient miser sur la langue française, d'autant que les communautés du Fleuve n'était pas sans avoir acquis quelque avance dans la maîtrise de cette langue par rapport à leurs co-religionnaires du nord.

C'est par ailleurs au nom même de la religion que les Bị̂tân jugeaient inacceptable la réticence prononcée d'une partie de la population à la promotion de la langue arabe. Puisque tous les Mauritaniens sont musulmans, pourquoi la langue de l'islam ne seraitelle pas la première langue du pays, celle que tous les enfants apprennent à l'école et qui leur sert de véhicule d'enseignement pendant toutes leurs études, celle qui est utilisée 
dans les médias et qui est employée comme langue de travail dans tous les établissements et services mauritaniens, publics comme privés?

Cet argument fut sans doute entendu et accepté avec une assez grande unanimité, au moins dans un premier temps, car la généralisation d'un enseignement coranique, en tout début de scolarisation, ne souleva guère de protestation quand elle fut instaurée, dès les premières années de l'indépendance.

Incontestablement, la langue arabe tire beaucoup de son aura et de sa force du fait qu'elle est la langue du Prophète, celle dans laquelle le message d'Allah a été transmis. En Mauritanie, l'islamisation de la population est non seulement généralisée, mais encore ancienne. Mais si l'on trouvait de bons lettrés dans les différentes communautés avant le $\mathrm{XX}^{\mathrm{e}}$ siècle, la colonisation française, puis l'indépendance, ont changé le statut de la langue arabe, ainsi que celui de la culture islamique traditionnelle dont elle était le véhicule attitré.

À partir des années 60, la langue arabe n'est plus uniquement la langue de la religion. C'est aussi — c'est d'abord, pour certains — la langue d'une grande communauté qui, de umma musulmane, est devenue une 'nation'. L'idée de nation arabe est surtout présente au sein des mouvements nationalistes d'obédience nassérienne et baathiste, mais elle s'impose également, quoique sous une forme un peu différente, dans les courants politiques influencés par le marxisme-léniniste ou le maoïsme.

$\mathrm{Au}$ total, on peut retrouver dans la plupart des courants politiques, qu'ils soient au pouvoir ou dans l'opposition, une représentation de la nation appuyée sur une unité/unification linguistique, fût-elle contrainte. La Mauritanie, à l'indépendance, a adopté le français et voulu construire un État sur le modèle jacobin, cherchant à dissimuler (et minimiser) au maximum les différences de pratiques langagières grâce à l'adoption de la langue des anciens colonisateurs, au moins pour une grande partie des usages officiels. Sous la pression de la majorité arabophone, elle s'est éloignée peu à peu de l'ensemble politique et économique formé par les pays francophones du Sud du Sahara et a progressivement attribué une place plus grande à l'arabe.

Durant toute cette période, le multilinguisme continue à être vu, moins comme une richesse que comme une tare à éradiquer, et le rêve du politique semble toujours peu ou prou d'avoir affaire à une communauté monolingue.

Cette attitude va, chez l'ancien président Moawiya ould Taya, jusqu'à l'oubli complet des communautés noires non arabophones de Mauritanie, et à la dénonciation du dialectal arabe - celui-ci étant considéré comme un facteur d'arriération, de sousdéveloppement culturel et économique, qu'il conviendrait de remplacer de toute urgence par l'arabe classique (Ould Taya, 2012). Comme l'a affirmé crûment Moawiya dans son livre consacré au « salut des Arabes » (Nažāt al-'arab) : la Mauritanie n'aurait pas connu tous ses problèmes si tout le monde avait parlé arabe.

Ce qui est malgré tout intéressant, dans l'affirmation très naïve de l'ancien Président de la R.I.M., c'est l'idée que les pratiques et les productions langagières devraient pouvoir être entièrement régulées. À vrai dire, d'autres avant lui ont été plus loin dans le souhait d'inventer une langue universelle, commune à tous les humains et ainsi susceptible de mettre fin à la malédiction divine - celle qui, selon le mythe de Babel, a condamné l'humanité à la dispersion et à l'incommunicabilité du fait de la multiplication des langues. L'échec des différentes tentatives montre bien cependant que, si l'on peut peser dans une certaine mesure sur les usages linguistiques - en particulier lexicographiques, 
mais pas seulement (cf. la renaissance planifiée de l'hébreu) —, il est très difficile de tout contrôler en ce qui concerne les langues et leurs usages.

Si l'on considère maintenant, non plus le statut de l'arabe par rapport aux autres langues nationales de Mauritanie, mais le cas de figure qu'il constitue par lui-même en tant que langue, on peut aussi se demander dans quelle mesure sa variation à travers l'espace et le temps est clairement perçue (et reconnue) par ceux qui la parlent.

Traditionnellement, en Mauritanie, l'existence de l'arabe standard était fondamentalement celle d'une langue écrite : un arabe 'littéral' (et littéraire) pour lequel le corpus de textes anciens — copié, recopié et mémorisé à l'envi — constituait un corset de références garant de la bonne orthodoxie.

Ces dernières années, la notion de diglossie a fait l'objet de nombreuses critiques dans son application au monde arabe, et il est vrai que le succès de ce concept, après que Ferguson l'a revisité en en proposant une lecture plus pragmatique mais aussi plus hiérarchisée, s'est souvent fait au détriment d'une vision plus objective de la réalité langagière (sans doute moins diglossique que triglossique, voire clairement 'métissée' - cf. ci-dessous). Pour la Mauritanie, cependant, la notion de diglossie telle que l'avait décrite précédemment Marçais - comme opposition de deux variétés apparentées, l'une écrite ('littérale') et l'autre orale — s'applique très bien à la situation de la société maure traditionnelle (Taine-Cheikh 2012). Cela fut, à l'époque de son âge d'or, un atout majeur dont l'œuvre de Aḥmad b. al-Amīn al-Šinqīṭi (al-Wasìt fi tarāžimi 'udabā'i šinqìt) peut porter témoignage. Mais quand il fallut faire de l'arabe littéral une langue de travail et d'enseignement, le chemin vers un nécessaire renouveau linguistique s'est trouvé entravé par les habitudes et les formations trop respectueuses de la doxa coranique et de son impératif catégorique selon lequel toute innovation est condamnable.

Que l'on considère, actuellement, que l'arabe doive servir pour enseigner des matières comme la philosophie et l'histoire, mais doive céder la place à une langue étrangère pour l'enseignement poussée des sciences et des techniques, c'est peut-être, de la part des dirigeants, faire preuve de pragmatisme, mais c'est sans doute aussi privilégier les usages idéologiques de l'arabe au détriment d'une appréhension plus globale de la langue. À ce propos, il est permis de penser que le conflit inter-ethnique autour de la politique linguistique aurait sans doute été moins aigu si celle-ci n'avait pas été instrumentalisée pour servir les intérêts de groupes particuliers et si les difficultés inhérentes au multiculturalisme et au plurilinguisme avaient été traitées avec moins de passion et plus d'objectivité scientifique.

\section{Le ḥassāniyya, dialecte... et langue?}

Parmi les données de base, il me semble qu'il aurait fallu tenir compte du fait qu'une partie de la population, celle des Bị̣̂̂an, avait pour langue maternelle un dialecte arabe, le ḩassāniyya, non pas (ou pas seulement) pour justifier l'adoption de l'arabe (standard) comme langue première de la nation toute entière, mais d'abord pour reconnaître que, en fonction de leur langue maternelle, les différentes communautés ethniques composant la Mauritanie se trouvaient dans une situation inégale face à l'acquisition de l'arabe. N'aurait-on pas pu favoriser des apprentissages différenciés et mettre en place des mécanismes régulateurs pour contrebalancer l'inégalité originelle d'accès à l'arabe comme langue d'enseignement ? N'était-il pas particulièrement contre-productif 
d'utiliser certaines matières, telle l'instruction civique et religieuse, comme variable d'ajustement et de sélection?

Curieusement, le hassāniyya a été l'objet, dans la seconde moitié du $\mathrm{XX}^{\mathrm{e}}$ siècle, d'une forme de déni qui n'a sans doute pas facilité l'intérêt des pédagogues et des décideurs à son égard. Francis de Chassey, lors des journées anniversaire du 28 novembre 2010, m'a rappelé la difficulté qu'avaient rencontré les étrangers, ainsi les coopérants français, à suivre un enseignement en hassāniyya au tout début des années 60 : c'était l'arabe standard qu'il fallait apprendre, pas le dialecte ! La consigne venait de très haut... et, sauf erreur, le hassāniyya ne fut jamais enseigné en Mauritanie que par l'intermédiaire des missions catholiques (du diocèse de Nouakchott notamment) ${ }^{2}$.

Pourtant, dans le même temps, j'ai plus d'une fois recueilli des manifestations de défense du dialecte et demeure convaincue que le hassāniyya est un élément fondamental de l'identité maure (bị̣̂̂niyyä). Le témoignage de David Cohen, auteur d'une remarquable étude sur 'la hassānīya' parue en 1963, est particulièrement symptomatique à cet égard. Celui dont je fis la connaissance à l'E.P.H.E. en 1974, et qui devint mon maître dans le vaste domaine de la dialectologie arabe et du chamitosémitique, avait gardé un souvenir ému et impressionné de ses conversations avec les étudiants mauritaniens à Paris qu'il avait croisés et qui lui avaient servi d'informateurs. Il me répéta souvent que ceux-ci n'étaient pas comme les autres arabophones qu'il avait rencontrés, car ils étaient attachés à leur dialecte et en défendaient la richesse. Il évoqua souvent devant moi son ami Mohammed ould Maouloud ould Daddah qui l'avait étonné en écrivant des poèmes en arabe sur les nappes en papier des restaurants (ayant contribué à la rédaction du livre, notamment en transcrivant les enregistrements, il apparaît comme collaborateur sous le nom de plume de Mohammed el Chennafi) ${ }^{3}$. Bien plus tard, en lisant ses mémoires, j'ai découvert que David Cohen avait également bien connu Moktar ould Daddah qui fut son condisciple à l'École des langues orientales. Le futur premier Président de la Mauritanie lui avait même fourni des matériaux, mais n'avait pas accepté de réaliser lui-même la description de son dialecte. Cohen précise à ce propos $(2008: 458)$ :

Son refus m'avait quelque peu surpris. Il aimait l'idiome qu'il parlait, il me récitait des poèmes qui, apparemment, le transportaient d'enthousiasme. Je notais les textes qu'il me récitait de temps en temps, mais il n'alla pas plus loin.

Les raisons de ses réticences m'apparurent clairement, peu de temps après. [...] Futur homme d'État arabe, il ne pouvait pas se compromettre, ni même cautionner, une étude d'arabe qui ne porterait pas sur la langue classique.

Ceci montre qu'une position officielle négative peut cacher, à condition qu'elle reste secrète, une attitude nettement plus favorable !

C'est après avoir commencé à travailler sur l'arabe 'médian' pour une thèse de $3^{\mathrm{e}}$ cycle, que David Cohen me confia les documents relatifs au dictionnaire d'Albert Leriche. Ce dernier, lors de son long séjour en Mauritanie dans les années cinquante, avait rédigé un Lexique Français-Hassāniyya que l'I.F.A.N. (en fait Théodore Monod, son directeur) semble avoir refusé de publier, pour une raison que j'ignore. Pourtant, plusieurs articles

\footnotetext{
${ }^{2} \mathrm{Ou}$, à une échelle infime, par les américains du Peace Corps.

${ }^{3}$ La disparition de Mohammed ould Maouloud (mort en septembre 2012) n'a précédé que de quelques mois celle de son vieil ami David Cohen (décédé en mars 2013).
} 
bien informés, notamment sur la musique et la poésie, étaient parus sous sa signature dans la revue de l'Institut ${ }^{4}$. Ce que je sus plus tard, c'est que David Cohen s'était rendu (avec Mohamed ould Maouloud ?) dans la famille de Leriche, après son décès, et avait dû donner une somme d'argent non négligeable en échange des manuscrits hérités du défunt pour que ce legs scientifique ne se perde pas définitivement sous la poussière d'un grenier.

Je n'avais encore travaillé que sur un tiers ou une petite moitié des données laissées par Leriche, quand l'opportunité de venir à Nouakchott à l'Institut Mauritanien de Recherche Scientifique se présenta en 1978. Il est probable que, sans la volonté d'Abdallah ould Babakar, alors directeur de l'I.M.R.S., de faire une place à l'étude du hassāniyya et à la rédaction du Dictionnaire hassāniyya - français (1988-1998), celuici n'aurait pas pu voir le début de sa réalisation.

À ce jour, le travail de rédaction reste inachevé, pour diverses raisons qui relèvent partiellement de mon fait, mais aussi des problèmes économiques rencontrés par Geuthner, ma maison d'édition (en effet, le neuvième volume n'a pas été imprimé).

Depuis le début de la publication, un certain nombre de dictionnaires de dialectologie arabe sont parus, venant enrichir la petite collection d'ouvrages réalisés avant ou juste après les indépendances, mais rares sont encore les études de ce genre réalisées par des arabophones (surtout si l'on se limite à ceux qui œuvrent dans leur pays d'origine).

Dans la mesure où la recherche porte naturellement sur ce qui pose problème et 'interpelle', il n'est pas surprenant de constater que, jusqu'à présent, les auteurs des dictionnaires, grammaires et atlas du monde arabe sont prioritairement des non arabophones. Dans le conflit — ou ce qui est généralement ressenti comme tel — entre l'arabe standard et le dialecte, c'est le premier qui semble devoir être promu, le second apparaissant soit comme indéfendable, soit comme suffisamment fort par lui-même pour qu'on n'ait pas besoin de prendre sa défense ${ }^{5}$.

Hormis le fait, indiscutable, que l'arabe standard a pour lui d'être une variété proche (ou très proche) de la langue dans laquelle le Coran a été révélé, deux autres arguments jouent en sa faveur : il peut être associé à une riche littérature écrite qui, pour partie, a traversé les siècles ${ }^{6}$ et il fournit un outil potentiel de communication entre les arabophones de tous les pays.

Les dialectes, quant à eux, ont généralement le défaut de présenter exactement les traits opposés. D'une part, ils ne sont pas associés à une littérature jugée digne de ce nom (c'est d'ailleurs le principal argument que Ferguson a donné pour légitimer une hiérarchie entre les deux glosses). D'autre part, ils présentent une variation si importante que certains dialectologues — je pense bien sûr en priorité à Behnstedt et Woidich, même si quelques atlas ont été dressés par d'autres avant eux - ont jugé que l'une des tâches les plus importantes consistait à établir des cartes avec, pour chaque trait (qu'il soit phonétique, morphologique ou lexical), la ou les localisation(s) géographique(s) de toutes les variantes recensées pour un espace donné.

\footnotetext{
${ }^{4}$ Leriche bénéficia, comme beaucoup d'autres, de la collaboration très éclairée de Mokhtar ould Hamidoun.

${ }^{5}$ La bibliographie des linguistes arabisants montre cependant que de nombreux dialectologues sont aussi des spécialistes de l'arabe classique, voir par exemple celle de Kees Versteegh, de Clive Holes ou de ElSaid Badawi.

${ }^{6}$ Il existe cependant toute une littérature sur le moyen-arabe qui montre que la variation existe aussi dans l'arabe écrit.
} 
Il arrive cependant que des points de vue divergents soient émis. Parmi les arabisants, Kallas (1999) est l'un de ceux qui se sont élevés le plus clairement en faveur du 'néoarabe' (terme qui renvoie à l'ensemble des dialectes arabes par opposition à la langue littéraire $)^{7}$. Sa défense du néo-arabe se fait au nom de la promotion de la variété orale (et 'maternelle') de l'arabe et sur la base de l'existence d'une littérature vivante, populaire, en arabe libanais. Elle est difficilement transposable ailleurs, cependant, car elle milite aussi pour une rupture prononcée avec l'héritage classique arabo-islamique dont seuls les Maltais ont jusqu'à présent fourni le modèle.

Sans forcément prôner un renversement aussi décisif, des voix se font souvent entendre pour évoquer telle ou telle variété d'arabe national (l'arabe 'tunisien', l'arabe 'libyen', l'arabe 'égyptien', l'arabe 'irakien'...) et pour suggérer que cette unité — sinon affirmée, du moins sous-entendue - devrait bénéficier d'une meilleure reconnaissance et, sans doute, d'un meilleur statut.

Il est clair que les réseaux de communication ont complètement changé ces dernières décennies, produisant des effets de convergence importants entre les différentes variétés d'arabe parlé. La centralisation des affaires publiques et des médias ainsi que les déplacements de population vers les régions les plus attractives n'ont eu de cesse de favoriser certaines réalisations linguistiques au détriment d'autres. Dans bien des cas, les traits sélectionnés ont été ceux d'un groupe particulier, parfois (mais pas nécessairement) parce qu'ils étaient considérés comme plus proches de l'arabe classique, souvent parce qu'ils correspondaient au parler des habitants de la capitale ${ }^{8}$.

La variation affectant l'arabe a-t-elle pour autant changé de nature ? Suit-elle dorénavant les limites dessinées par les frontières ? Ou bien est-ce l'attitude passéiste des dialectologues qui, à force de prêter attention au détail, crée de toute pièce des représentations de l'arabe variant à l'infini ?

Il est probable que l'une et l'autre hypothèses soient fausses dans l'absolu, tout en portant une part de vérité. Les dernières enquêtes au Maroc montrent par exemple que les habitudes linguistiques anciennes n'ont pas disparu du jour au lendemain à Rabat, Fès ou Casablanca (voir par exemple Hachimi 2011), mais qu'elles n'entravent guère la communication. Ce n'est sans doute pas uniquement un effet de la politique vis-à-vis des territoires du Sud anciennement colonisés par l'Espagne si le hassāniyya est actuellement, en dehors de l'arabe standard, la seule variété d'arabe reconnue comme langue nationale dans la constitution du royaume chérifien ${ }^{9}$.

Lorsque l'on étudie le hassāniyya comme je le fais depuis plusieurs décennies, on ne peut qu'être frappé par le cas de figure très original qu'il représente par rapport aux autres variétés d'arabe parlées de par le monde.

Globalement, on peut dire que cinq traits majeurs le définissent.

i) C'est un dialecte de type maghrébin (ce qui se perçoit notamment à sa structure

\footnotetext{
${ }^{7} \mathrm{Si}$ j'ai quelque réticence envers l'emploi de ce terme, c'est parce qu'il va souvent de pair avec une vision unifiante (et à mon avis réductrice) des dialectes, selon laquelle le néo-arabe serait de type analytique, par opposition à l'arabe classique de type synthétique.

${ }^{8}$ C'est le cas par exemple de l'Égypte où le parler du Caire a un rayonnement national (et même au-delà, grâce notamment à ses productions cinématographiques, télévisuelles et — plus récemment, maintenant que le dialecte tend à s'écrire - littéraires).

${ }^{9}$ Quoi que portant sur des discriminants précis, l'étude de Heath (2002) montre bien la spécificité quasi constante du hassāniyya par rapport aux autres variétés marocaine. Pour un aperçu sur la question historique et sociolinguistique du hassāniyya au Maroc, voir Taine-Cheikh 1997.
} 
syllabique pauvre en voyelles, à sa conjugaison — en particulier aux marques de $1^{\text {ère }}$ personne - et à certaines de ses particules).

ii) C'est un dialecte de type bédouin (ce qui se reconnaît à certains traits phonéticophonologiques comme la réalisation $g$ du $q \hat{a} f$ et la conservation des interdentales, cf. Taine-Cheikh 1999, mais aussi à une multitude d'autres traits qui, bien souvent, peuvent être résumés comme une tendance au conservatisme).

iii) C'est un dialecte à substrat berbère (ce qui transparaît clairement dans le lexique, mais a certainement joué aussi un rôle dans certaines innovations, telle la création d'une dérivation verbale factitive à préfixe $s a-$, cf. Cohen 1963 : 132-3 et Taine-Cheikh 2003a).

iv) C'est un dialecte auquel est associée une très riche littérature orale de nature essentiellement poétique (cf. le projet Traditions orales traditions écrites de Mauritanie de l'I.M.R.S.).

v) C'est un dialecte très unifié parlé sur plus d'un million de $\mathrm{km} 2$ (bien au delà des frontières de la Mauritanie — non seulement au Nord, mais aussi au Nord-nord-est et à l'Est).

Si la convergence observée ailleurs depuis quelques décennies est l'un des effets des changements socio-économiques affectant les sociétés traditionnelles, cette unification du dialecte hassāniyya est beaucoup plus ancienne... et beaucoup plus surprenante. Elle se manifeste dans le lexique, comme je l'ai toujours affirmé (cf. Taine-Cheikh 19881998 et 1991) et comme cela ressort aussi assez clairement des deux tomes parus du Wortatlas... de Behnstedt \& Woidich $(2011,2012)^{10}$. Elle se manifeste encore plus nettement à travers les innovations morpho-syntaxiques partagées (en tout premier lieu, on citera le passif en $u$ - des verbes dérivés, cf. Taine-Cheikh 1983, mais s'y ajoutent bien d'autres traits sur lesquels nombre de mes travaux ultérieurs ont mis l'accent).

Le caractère très homogène et si spécifique du hassāniyya confère à cette variété linguistique une autonomie et une dimension nationale que peu d'autres dialectes présentent au même titre. Elles lui vaudraient sans doute la qualification de 'langue' si ses locuteurs mauritaniens avaient à cœur de la revendiquer. Pourtant, autant qu'il m'en paraisse, cette idée est bien loin de régner chez les hassanophones, aujourd'hui encore moins qu'hier. Par le passé, le hassāniyya a certainement joué un rôle majeur dans la construction de l'identité bị̣̂aniyyä et dans la formation de la culture bédouine, les différents traits le caractérisant se combinant pour qu'émergent et se transmettent des pratiques linguistiques appuyées sur un corpus poétique riche et vivant.

Aujourd'hui, les contours les plus spécifiques deviennent moins visibles, notamment en ce qui concerne le lexique où une partie du vocabulaire tend à se perdre, en même temps que les coutumes et objets matériels qu'elle nommait sont abandonnés. C'est pour tenter de les saisir avant qu'ils disparaissent tout à fait que je me suis tournée vers l'étude du berbère, à partir des années 97-98.

\footnotetext{
${ }^{10} \mathrm{~J}$ 'ai fait un commentaire plus détaillé et plus circonstancié des résultats concernant le hassāniyya lors de mon intervention à la journée d'étude ("Le Maghreb à travers les dictionnaires : pratiques linguistiques et imaginaires socio-culturels. Approches comparatistes ») organisée par le LACNADCREAM à l'INALCO, en juin 2013.
} 


\section{Le zénaga, par delà le décès prévisible}

De fait, mon premier contact rapproché avec le berbère, et plus précisément, avec le zénaga, date de 1978. En effet, peu après mon arrivée à Nouakchott, Mokhtar ould Hamidoun vint un jour déposer sur la table d'Abdel Wedoud ould Cheikh, mais à mon intention $^{11}$, des dossiers contenant quelques centaines de pages manuscrites. Il souhaitait que je les étudie et dise si ces documents méritaient d'être publiés en arabe.

J'avais dans ma bibliothèque l'ouvrage sur le zénaga paru dans la collection de l'I.F.A.N. en 1953. Je procédai alors à une comparaison page par page et constatai avec beaucoup de surprise que le texte imprimé, publié sous le nom de Francis Nicolas, suivait pratiquement ligne à ligne celui écrit de la main de Mokhtar. Un certain nombre de signes permettaient d'affirmer que le manuscrit était bien le texte originel : non seulement les retours à la ligne du texte imprimé ne s'expliquaient souvent que par la disposition du texte manuscrit (et notamment la largeur parfois réduite de la feuille de papier utilisée), mais encore on pouvait clairement identifier nombre de variantes du texte imprimé à des erreurs de lecture ou de frappe, le texte manuscrit étant dans l'ensemble plus cohérent que le texte édité. La conclusion que j'en tirais alors — et faisais transmettre à Mokhtar ould Hamidoun — était qu'il fallait écrire un article rectificatif, avec la liste des principales erreurs contenues dans l'ouvrage de l'I.F.A.N. (telles qu'une simple comparaison des deux textes permettaient de le faire). Mais il me semblait surtout important de dénoncer ce que je considérais comme une imposture, en montrant quel était le véritable auteur. En retour, Mokhtar ould Hamidoun me fit savoir, sans plus de précision, qu'il ne tenait pas à soulever cette question et la chose en resta là. Durant les 16 années que je passai à l'I.M.R.S., mes quelques tentatives pour travailler avec un informateur parlant zénaga n'aboutirent guère. Ridwan Collins, un Américain naturalisé marocain à la retraite, vint faire un premier travail de repérage dans les années 80 . Bon berbérisant ${ }^{12}$, il me dit combien le parler berbère de Mauritanie lui paraissait digne d'intérêt. Il évoqua son originalité et souligna deux conservatismes qui l'avaient frappé durant sa brève enquête : l'existence de termes berbères, d'une part pour désigner "la mer, la rivière" $(\partial L)$, d'autre part, pour compter ${ }^{13}$. Malheureusement, sa disparition accidentelle dans les montagnes de l'Atlas, au printemps suivant, l'empêcha de revenir en Mauritanie et de réaliser son projet.

Je ne repris le flambeau qu'à l'été 1997 et, grâce à la rencontre de Mohameden ould Ahmedou Yahya, il m'a été permis, depuis, de faire grandement avancer l'étude du zénaga.

À cette occasion, les données contenues dans les manuscrits de Mokhtar et dans le livre 'de' Nicolas furent passées au crible, et j'eus ainsi l'opportunité de relever notamment dans des notes de bas de page ou dans des comparaisons avec les parlers touaregs du Niger - les initiales «F. N.» qui ne pouvaient être interprétées que comme les initiales de «Francis Nicolas ». Par ailleurs, je finis par trouver, dans les archives de Mokhtar, une lettre signée de ce même Francis Nicolas qui recommandait à

\footnotetext{
${ }^{11}$ Nous partagions alors le même bureau à l'I.M.R.S. et la saḥwä empêchait donc Mokhtar de s'adresser à moi directement.

${ }^{12}$ Il a publié notamment deux articles remarqués sur le berbère de Tunisie (1981 et 1982).

${ }^{13}$ Pour une étude des numéraux en zénaga et une comparaison détaillée avec les systèmes berbères les plus conservateurs, voir Taine-Cheikh 2005.
} 
la publication la grammaire de Mokhtar ould Hamidoun. Ceci confortait l'annonce, faite dans l'« Avant-propos» au Précis sur la Mauritanie, de la parution prochaine d'un ouvrage du même auteur sur le zénaga ${ }^{14}$. Indéniablement, cela signifiait que l'attribution de la publication à Francis Nicolas avait dû se décider très tardivement. Avait-elle pu se faire à l'insu de Mokhtar ould Hamidoun ? Cela me sembla impossible, même si l'apport de Mokhtar, en tant que collaborateur de l'I.F.A.N. (puis, plus tard, comme chercheur à l'I.M.R.S.), ne fut pas toujours reconnu à sa juste mesure, et même si Mokhtar avait incontestablement l'habitude de distribuer très largement son savoir (jusqu'à ses notes, soustraites souvent trop généreusement de ses archives personnelles). J'acquis donc la conviction que Mokhtar avait pu jouer un rôle actif dans l'attribution de son travail à Nicolas et que, loin d'avoir été la victime d'une usurpation ou d'un plagiat, il avait pu trouvé satisfaisant d'avoir un prête-nom.

Avait-il effectivement travaillé avec Francis Nicolas pour une partie ou l'autre de l'ouvrage $?^{15}$ A-t-il pu penser que l'ouvrage aurait plus de chance d'être édité s'il portait le (seul) nom de Nicolas - auteur ayant déjà publié un ouvrage sur le berbère dans la collection I.F.A.N. ? Ces hypothèses ne sont pas à exclure, mais ce qui me paraît le plus vraisemblable est l'hypothèse que je présentai pour la première fois au XII ${ }^{\mathrm{e}}$ congrès de l'A.F.E.M.A.M., en 1998, dans une intervention intitulée "La berbérité en question : le cas d'un auteur "masqué »"16. À mon avis, Mokhtar ould Hamidoun a choisi délibérément de ne pas attacher son nom à la recherche sur le zénaga, de ne pas se faire le porte-drapeau d'une culture en voie de disparition, de peur sans doute que cela n'entrave un peu plus son principal objectif de recherche : l'histoire des tribus maures.

Dans les années 80, plusieurs événements (disparition mystérieuse d'une thèse, agression violente d'une jeune enseignante de l'Université, après qu'elle eût fait imprimer à l'étranger un manuscrit avec ses propres notes d'édition) donnèrent à penser que certains membres de la société civile surveillaient de très près les chercheurs, dès lors que ceux-ci prétendaient écrire objectivement sur leurs ancêtres et leurs statuts sociaux. Depuis, la pression qu'elle n'a cessé d'exercer pour contrôler l'écriture de l'histoire n'a fait que grandir ${ }^{17}$. Mais on aurait tort de penser qu'elle n'a commencé que dans ces années-là, car Mokhtar lui-même eut à souffrir des conséquences de ses recherches : il fut agressé dans sa jeunesse et si sérieusement menacé pour ses écrits que cela semble avoir quelque peu influé sur ses envies de publier (ou du moins de 'tout' écrire) $)^{18}$.

Dans le contexte de l'indépendance, le zénaga n'avait pas bonne presse, et même si la diminution inexorable du nombre de locuteurs et l'absence de revendications identitaires ne faisaient de lui aucunement une menace objective pour l'arabité' des Bị̣̂̂n, le

\footnotetext{
${ }^{14}$ Les auteurs de l'« Avant-propos », Duchemin et Leriche, évoquaient (p. 6) les informations fournies au berbérisant Francis Nicolas, mais aussi le travail personnel de Mokhtar ould Hamidoun sur "son propre terroir, dernier îlot berbérophone du Sahara occidental, au dialecte duquel il a consacré une longue étude qui paraîtra, espérons-le, bientôt".

${ }^{15}$ Récemment m'est parvenue la partie grammaticale de l'ouvrage, écrite cette fois de la main de Francis Nicolas. Apparemment, ce texte fut confié par l'auteur à Jacob Oliel.

${ }_{17}^{16}$ Pour plus de détails, voir l'« Introduction » au Dictionnaire zénaga - français (2008 : LX-LXI).

${ }^{17}$ C'est d'ailleurs un des problèmes majeurs de la recherche en Mauritanie (cf. Ould Cheikh 1999).

18 J'ai moi-même ressenti les effets délétères de la censure sociale (et tribale), à propos du diwân de Muhammad wuld Äbnu wuld Ḥmaydä — une publication préparée par Ahmed ould Habiboullah (dans le cadre du projet Traditions orales traditions écrites de Mauritanie de l'I.M.R.S.) qui ne put de ce fait aboutir.
} 
prendre comme objet d'étude devait être encore plus mal vu que d'étudier le hassāniyya. Le faire sans l'assumer publiquement, en affichant son nom sur la page de garde, pouvait donc bien être dans la manière däymâniyyä, une façon retenue de se comporter tout autant qu'une façon détournée de dire les choses ${ }^{19}$.

Cependant, en ce qui concerne les travaux de Mokhtar sur le zénaga, on ne peut affirmer que ses manuscrits ou le 'pseudo Nicolas' pêchent par allusion (ils pêchent plutôt par l'excès - excès de variations notamment), sauf à considérer que l'absence d'une certaine rigueur dans la transcription et la description linguistique ait pu être autre chose que le produit d'une formation scientifique insuffisante. Le faible écho qu'eut le 'pseudo Nicolas' auprès des berbérisants — en dehors de quelques comparatistes comme Maarten Kossmann (1999) — tient au fait que le système phonétique et phonologique de la langue restait très impénétrable et d'autant plus difficile à saisir que sa logique était passablement obscurcie par la transcription choisie pour certains sons (ceux-là même qui apparaissaient dans les mots dont la notation tendait à varier d'une page à l'autre).

Mais l'accueil n'aurait sans doute pas été aussi discret si le zénaga ne s'écartait pas autant des autres parlers berbères connus. Pour les auteurs comme Aikhenvald (1988), qui avaient pour but avoué de proposer une classification des parlers berbères, la situation était claire : le zénaga constituait un groupe à lui tout seul (le groupe " ouest»), tout comme le touareg (le groupe " sud»), tandis que les parlers septentrionaux se répartissaient entre le berbère "de l'est " (parlers de Libye — à l'exception de celui de Zouara - et d'Egypte) et le berbère « du nord » (les parlers du Maroc, d'Algérie et de Tunisie, ainsi que celui de Zouara).

Mes recherches sur le zénaga n'ont pas infirmé ce classement, et les travaux récents de d'Attayoub et de Lux sur la tetserret du Niger n'ont fait que confirmer l'existence ancienne d'une variété ouest-saharienne, globalement distincte des variétés touarègues. En effet, la tetserret, langue berbère en voie de disparition des Ayttawari Seslem ${ }^{20}$ qui n'est pas compréhensible pour les locuteurs du tamacheq — présente des caractéristiques (notamment phonologiques) dont certaines ne sont communes qu'avec le zénaga (Lux 2011, 2013). Si l'on ajoute à cela les résultats des travaux de Lameen Souag sur le kwarandzyey (parler mixte berbèro-songhay de Tabelbala, au sud de

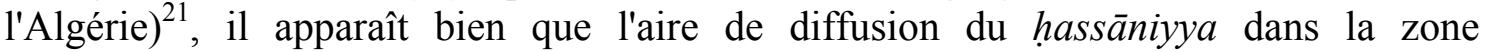
saharienne ouest-africaine a été précédée, à une époque plus ancienne, par une aire (très étendue également) où l'on parlait, ou du moins était en contact, avec une variété de berbère proche ou identique au zénaga.

L'aire d'extension maximale de la tuzzungiyyä est difficile à préciser, mais il est vraisemblable que cette variété de berbère, depuis longtemps réduite à une région très limitée du Sud-Ouest de la Mauritanie, a couvert par le passé l'ensemble des régions nord et est de ce pays. J'en ai voulu pour preuve l'existence, dans la toponymie, d'un nombre important de noms d'origine berbère dont la forme atteste la présence des affixes particuliers du diminutif. En effet, alors qu'en berbère, le féminin des noms tend

\footnotetext{
${ }^{19}$ Cf. le Klâm äwlād Däymān, recueil d'anecdotes illustrant le comportement maddäymânn. Ce travail a été réalisé par Ḥusayn ibn Maḥand, le propre petit-fils de Mokhtar, dans le cadre du même projet T.O.T.E.M.

20 L'inégalité du bilinguisme tetserret-tamacheq est comparable à celle du bilinguisme zénagahassāniyya (cf. Walentowitz. \& Attayoub 2001).

${ }^{21}$ Voir notamment son article de 2010 .
} 
à servir de diminutif au masculin (le masculin servant parfois, quant à lui, d'augmentatif au féminin), le zénaga fait exception avec un système de dérivation original : les nominaux masculins forment leurs diminutifs, au singulier, par préfixation de $a \dot{g}$ - et suffixation de $-t$, et les nominaux féminins, par le simple ajout du préfixe $t^{y} \bar{a}^{22}$. Malgré l'évolution fréquente de $a \dot{g}$ - en $a g$ - ou même $a k$-, on pourra donc reconnaître assez facilement des diminutifs dans des toponymes comme Akjoujt (cf. zénaga äžžäwž "trou" et son diminutif $a \dot{g} \underline{z} \ddot{a} w \underline{z} t)$. Comme je l'écrivais dans l'«Introduction» à mon Dictionnaire zénaga-français (2008 : XLIX) :

«Il est très intéressant de constater que l'on trouve un Aghlenbit à $35 \mathrm{~km}$ au S.-O. de Tidjikja, un Aghenghest au nord de Oualata et un certain nombre de petites dépressions de l'est de la Mauritanie dont le nom, sur les cartes, commence pas Aghiert (ainsi zén. awyär "dépression argileuse salée" > dim. agawyärt).

La dérivation diminutive en $a \dot{g} . . . t$ n'étant pas attestée en dehors du zénaga, nous avons là des indices sérieux, me semble-t-il, d'une présence ancienne du zénaga dans une grande partie de la Mauritanie actuelle, y compris dans la partie orientale du pays où les domaines respectifs du zénaga et du touareg ne sont pas évidents a priori à délimiter. »

En effet, à l'échelle de l'ensemble du berbère, les ressemblances avec le touareg ne sont pas non plus négligeables, en particulier au plan lexical, et même si la tetserret présente des spécificités remarquables avec le zénaga - notamment par la richesse de son système vocalique, par sa tendance à l'amuissement de $\dot{g}(>?$ en zénaga et $>\varnothing$ en tetserret) ou encore par la nature et le comportement de la marque de $1^{\text {ère }}$ personne du singulier en $-g / k-$, il y a aussi une proximité plus grande de toute l'aire berbère méridionale qui s'étend même à ses frontières, tant la tachelhit marocaine d'un côté que le ghadamsi libyen de l'autre.

Lorsque l'on procède à des comparaisons à l'intérieur d'un ensemble de parlers distincts relevant d'une famille de langues unique, les rapprochements et les différences que l'on peut mettre à jour sont loin d'être simples et de converger vers une conclusion unilatérale. Il est impossible ici de faire un bilan exhaustif, tout juste signalerai-je un cas où les données du zénaga sont plus proches des parlers septentrionaux que des parlers méridionaux : celui qui concerne la tendance à la 'spirantisation' des occlusives en position intervocalique $(d>d, d>d \ldots)^{23}$. Jusqu'à mes travaux sur le zénaga, ce phénomène était considéré comme une particularité de certains parlers du nord, à l'exclusion absolue de ceux du sud.

L'un des enjeux de l'étude du zénaga, au-delà de la description d'une langue menacée de disparition, est de distinguer les innovations des conservatismes, les développements parallèles des traits anciens du proto-berbère. Pour tout cela, le zénaga est d'un apport

\footnotetext{
${ }^{22}$ Pour la formation des pluriels et pour une hypothèse sur la morphogénèse de ces diminutifs, cf. Taine-

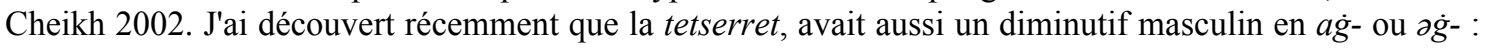
ex. agmajgar "petit esclave", diminutif de majgar "esclave" (Attayoub 2001 : $46-j$ notant chez cet auteur la chuintante $\check{z}$ ). C'est un argument de plus en faveur de l'existence d'une variété de berbère ouestsaharienne, mais il existe une différence notable par rapport au zénaga : l'absence du suffixe -t. Il pourrait s'agir cependant de l'effet d'une évolution tardive - comparable à celle qui pourrait avoir affecté le diminutif féminin (dorénavant en $t$ - comme tout nom féminin singulier).

${ }^{23}$ Le zénaga présente cependant des spécificités tout à fait remarquables dans la manière de mettre en œuvre cette 'spirantisation', telles les évolutions $[\mathrm{t}]>[\mathrm{d}],[\mathrm{z}]>[\mathrm{t}]$ et $[\mathrm{z}]>[\mathrm{t}]$ (Taine-Cheikh 1999 et 2003b).
} 
considérable. Mais, outre son intérêt pour la linguistique historique des langues en général et du berbère en particulier, il n'en est pas moins important pour l'histoire culturelle et sociétale des Bīđân et, plus largement, pour celle de la région.

\section{Mythes et autres idées reçues}

La connaissance avance par accumulation, mais elle avance aussi par tâtonnement et en examinant d'un œil critique ce qui se répète depuis la nuit des temps. Parmi les approximations, demi vérités et réelles erreurs qui touchent à mon champ de recherche, toutes ne concernent pas le même public et ne présentent pas le même intérêt.

Par exemple, je crois inutile de m'attarder longuement sur une affirmation telle que "les dialectes n'ont aucune règle et aucune logique", même si, de toutes les déclarations péremptoires de Moawiya ould Taya, celle-ci est sans doute l'une des idées les plus répandues en Mauritanie et, plus largement, dans le monde (arabe notamment). L'activité linguistique vient justement démontrer, par l'élaboration de dictionnaires et surtout de grammaires, que toutes les variétés langagières obéissent à des règles, qu'elles se pratiquent à l'oral ou à l'écrit, et qu'elles soient nationales, régionales ou locales. En hassāniyya, les passifs des verbes quadrilitères et des formes dérivées actives se forment tout aussi régulièrement qu'en arabe classique. Que la dérivation soit plus simple dans le dialecte mauritanien (où le passif se forme simplement et uniformément par préfixation d'un $u$ - $)^{24}$ ou que le nom d'action de la seconde forme (à $2^{\mathrm{e}}$ radicale géminée) soit de schème təf $f^{\uparrow} \hat{a} l$ et non de schème taf ${ }^{\complement} \bar{l}$ (ex. hass. təždâd vs cl. taždi d "renouvellement") ne veut pas dire qu'il n'y a pas de règle, mais seulement que celle-ci est différente.

Fondamentalement, ce sont les règles phonético-phonologiques, morphologiques et syntaxiques qui structurent l'usage de la langue. Ces règles concernent également le lexique, mais c'est dans ce domaine, bien entendu, qu'on observe le plus de variété.

En hassāniyya, les variations lexicales sont très limitées tant qu'on reste dans le vocabulaire usuel relatif à l'ensemble de la société traditionnelle. C'est là un fait d'autant plus remarquable qu'il s'agit d'un dialecte parlé sur un territoire très étendue, où les locuteurs se trouvent en contact depuis toujours avec des parlers divers, en Mauritanie même comme à l'extérieur des frontières (arabe marocain au nord, arabe algérien au nord-nord-est, touareg à l'est, bambara et soninké au sud-est, pulaar au sud, wolof au sud-ouest, sans compter les situations d'émigration plus lointaine).

Les particularités lexicales augmentent, bien entendu, dès lors qu'il s'agit de champs sémantiques spécifiques, tels ceux concernant les mines de sel et les palmiers-dattiers ou la musique, la chasse et la pêche.

Anciennement, les activités de pêche et de chasse relevaient, comme celles de musique ou d'artisanat, du domaine peu ou prou réservé de certains groupes particuliers de la société : les imrâgan d'une part, les nmâdi d'autre part. Au cours du $\mathrm{XX}^{\mathrm{e}}$ siècle, ces deux dernières communautés furent l'objet d'un traitement très particulier dans la littérature orientalisante et ethnologique, et cela eut des conséquences directes sur la manière dont ils furent considérés, eux et leurs particularités linguistiques. En effet, à force de mettre

\footnotetext{
${ }^{24}$ Contrairement à ce qui se passe en arabe classique, la vocalisation en $u$ (ici en gras) n'est jamais attestée à l'actif en hassāniyya. Comparer, pour "changer, renouveler", l'actif (hass. žäddäd/ižäddäd vs cl.

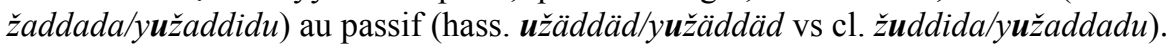


l'accent sur leurs spécificités, les apprentis-ethnologues ont fait croire aux linguistes mal informés qu'il existait en Mauritanie trois langues - et autant d'ethnies - menacées de disparition. Le travail de recensement des langues en danger est un travail utile et difficile à mener, certains territoires pouvant concentrer des dizaines, voire des centaines de langues dont la survie est souvent menacée en quelques années, surtout à l'époque présente de mondialisation/glocalisation forcenée ${ }^{25}$. Il est malgré tout fort regrettable que des contre-vérités apparaissent dans un ouvrage censé faire référence comme l'Atlas of the World's Languages in Danger de l'Unesco. Cet atlas, qui a été établi sous la direction de Moseley et dont une version interactive est accessible par internet à l'adresse suivante www.unesco.org/culture/en/endangeredlanguages) affirme en effet (p. 29) :

« In Mauritania, Zenaga, Nemadi and the language of Imeraguen are definitely endangered and may become critically endangered if the current social and cultural stigma attached to them is maintained and the languages continue to be excluded from the public sphere ».

Les mêmes informations étant également présentes dans l'article de Blench $(2007)^{26}$, il m'a semblé important de rétablir la vérité sur la question. Dans l'article "Des ethnies chimériques aux langues fantômes. L'exemple des Imraguen et Nemâdi de Mauritanie" (Taine-Cheikh 2013), je me suis donc attachée à démontrer que, si le zénaga était effectivement une langue menacée (mais une langue probablement 'sans ethnie' — du moins si l'on se réfère à l'absence de référents identitaires s'en revendiquant), on ne pouvait pas en dire autant des pseudo-langues imrâgən et nmâdi. L'existence de lexiques spécifiques ne suffit pas, en effet, pour conclure à l'existence de langues autres que le hassāniyya, même si de nombreux emprunts (notamment au zénaga) ont pu être relevés. Par ailleurs, si ces lexiques spécifiques sont composés en bonne partie de lexèmes d'origine berbère (généralement zénaga, mais pas uniquement) ${ }^{27}$, ils comptent aussi un grand nombre des lexèmes d'origine arabe, que la forme et surtout le sens soient identiques ou différents de celui qu'ils ont en ḩassāniyya. Voici quelques exemples de termes du hassāniyya employés par les Némadi avec un sens spécifique (Taine-Cheikh $2013: 158)$ :

\begin{abstract}
$\check{z} d i$ « jeune gibier » vs « chevreau, cabri »; hazmä « paquet de viande comprenant cinq gibiers »vs « liasse, fagot, botte, paquet »; ddällä (nom d'action tad’lyä) « revenir de la chasse (gäymârä) » vs «pendre ; descendre de chameau en se laissant glisser le long de la patte avant»; mrașși «(chien) qui, pendant les premiers jours de la gäymârä, a perdu l'habitude de courir»vs «s'arrêtant (pour un véhicule)»; maṣdar « départ du campement avec l'eau pour un certain nombre de jours $(30,40, \ldots)$ » vs « retour d'un point d'eau, départ du puits».
\end{abstract}

Si l'existence d'ethnies ou de langues imragen et nmâdi relève incontestablement de

\footnotetext{
${ }^{25}$ Les travaux de mes collègues du Lacito sont très instructifs à ce sujet — voir notamment ceux d'Alexandre François au Vanuatu.

${ }^{26}$ L'article est paru dans Language Diversity Endangered, un ouvrage à l'intention des spécialistes publié par le très sérieux éditeur Mouton de Gruyter.

${ }^{27}$ Le nominal gäymârä "chasse hauturière, au gros gibier, pratiquée avec des chiens" et le verbe correspondant gäymär sont une illustration du cas a priori surprenant (mais, de fait, non isolé) où l'équivalent ne semble pas (plus ?) attesté en zénaga, mais se retrouve dans d'autres parlers berbères. En l'occurrence, il s'agit de la racine pan-berbère GMR qui a donné, par exemple, $g m r / g^{w} m r$ "pécher ; chasser" en chleuh et gmer "cueillir" en kabyle (Naït-Zerrad 2002 : 809).
} 
constructions imaginaires, il ne s'agit pas de mythes à large diffusion. En effet, chez les linguistes spécialistes du domaine, les représentations les plus courantes - relativement aux productions langagières locales — consistent surtout, me semble-t-il, à surestimer quelque peu les effets du contact. Ainsi, de la proximité géographique, économique et culturelle, des Bị̂tân avec les populations noires non arabophones du Sahel, beaucoup de personnes - y compris parmi les arabisants — en ont conclu que les spécificités linguistiques du hassāniyya étaient dues à des emprunts très importants de l'arabe mauritanien aux autres langues du pays. De même, par un raisonnement assez comparable, les berbérisants ont souvent eu tendance à penser que le hassāniyya était responsable, non seulement du recul inexorable du zénaga, mais encore de ses nombreuses particularités linguistiques ${ }^{28}$.

C'est notamment pour contester ces visions, qui me paraissent faussées par des extrapolations à partir de faits extrêmement circonscrits (tel l'usage du terme mbûru au lieu de hubz pour "pain"), que j'ai cherché à mesurer les effets du contact sur la genèse des variétés arabe et berbère de Mauritanie. J'ai ainsi montré, dans "Périphérie géographique et perméabilité aux contacts. Le cas du Maghreb" (2007), que la position périphérique du domaine du hassāniyya n'était pas à interpréter comme un facteur automatique de divergence linguistique, du fait notamment du conservatisme particulier aux parlers de nomades. J'ai aussi étudié, dans "Arabe(s) et berbère en contact : le cas mauritanien" (2008), toutes les particularités qui pouvaient être imputables au contact, et montré que, si les phénomènes d'évolution parallèle ne manquaient pas, rares étaient les innovations morphosyntaxiques et même phonologiques qui se laissaient réduire à une influence directe extérieure.

Le lexique fournit bien sûr beaucoup plus de cas d'emprunts directs (du zénaga au hassāniyya et du hassāniyya au zénaga), mais il fournit aussi des cas de calques ainsi que bon nombre de néologismes d'origine obscure. Parallèlement à mes travaux proprement lexicographiques, j'ai entrepris d'étudier plus spécifiquement certains champs sémantiques (Taine-Cheikh 2010a et à paraître). Cela m'a permis de faire ressortir les nombreux échanges et parallélismes sémantiques entre le zénaga et le hassāniyya. Cela m'a amenée aussi à souligner la richesse relative du berbère mauritanien dans des domaines où il était réputé (très) pauvre, sous prétexte que le hassāniyya lui avait moins emprunté dans ce champ que dans d'autres (tel le domaine de l'élevage camelin par rapport à celui de l'élevage bovin $)^{29}$.

Ces recherches devraient contribuer à nuancer l'image traditionnelle que la société Bịđân se fait du zénaga. La complexité des phénomènes linguistiques et la nécessaire technicité à laquelle on doit recourir pour en rendre compte avec la plus grande précision obscurcissent cependant, à n'en pas douter, la lecture des faits qu'on aurait voulu claire et limpide. Il me semble toutefois que le (la) linguiste n'est pas seul(e) en cause. Parfois, certaines interprétations sont si ancrées dans les traditions orales ou écrites mauritaniennes qu'il semble impossible de convaincre un Mauritanien que la vérité scientifique puisse être autre que l'idée dominante qui circule dans la société.

\footnotetext{
${ }^{28}$ Pour Chaker, c'est l'influence des langues voisines non afro-asiatiques sur le zénaga qui est marquant. Il l'estime supérieure à celle du haoussa sur le touareg (qu'il juge déjà très importante) : « Le cas du zénaga de Mauritanie est encore plus évident sur ce plan des probables influences négro-africaines » (2012 : 112, note 6).

${ }^{29}$ Mon Dictionnaire français - zénaga (Taine-Cheikh 2010b) qui fournit un accès aisé au lexique zénaga par les entrées du français, permet aussi de voir quels sont les champs sémantiques les plus riches.
} 
Le premier exemple de ce type me semble être représenté par le couple $(\bar{a})$ żnâgä / șanha(d)ža. Alors que tout le monde est d'accord pour considérer que ces deux termes ont une origine commune, les lettrés mauritaniens sont persuadés que le second procède du premier, tandis que les linguistes quelque peu informés des réalités du berbère et de l'arabe assurent que c'est au contraire le second qui constitue une version arabisée du premier (Colin 1930 : 104-120). L'existence de ce doublon est un effet direct de la diglossie, mais on n'aboutit à deux formes distinctes perçues comme autonomes — l'une issue de l'oral et l'autre issue de l'écrit en arabe littéraire - qu'au terme d'une 'auralisation' de la forme écrite (sur la notion d'auralisation', cf. Taine-Cheikh 1998) ${ }^{30}$. Le second exemple concerne l'origine soi-disant himyarite des Berbères. Cette affirmation, qui s'applique également aux zénagophones de Mauritanie, s'appuie sur les écrits d'Ibn Khaldūn, mais elle n'est étayée par aucun fait patent et Ibn Khaldūn luimême n'y accordait pas foi (cf. Robin 2000 : 3473). Au contraire, tout ce que l'on sait des langues du sud de la péninsule arabique, et en particulier de l'himyarite, ne permet aucunement de penser qu'elles peuvent avoir un lien avec la famille des langues berbères. Etablir une relation de parenté entre les Berbères et les gens du sud de la péninsule revient donc essentiellement à assimiler fictivement les non-Arabes d'Afrique du nord (et du nord-ouest) aux non-Arabes d'Arabie.

\section{Pour conclure}

Faire le bilan, pour la Mauritanie, de cinquante années de recherche en linguistique n'est pas sans poser un problème. D'un côté, j'ai l'impression que, même si je n'ai commencé à travailler qu'une dizaine d'années après l'indépendance, il m'est difficile de rendre compte en quelques pages de mes recherches et de l'ensemble des résultats obtenus. Je n'ai pas évoqué ici mes travaux sur la poésie et n'ai guère détaillé ceux sur les noms propres (toponymes et anthroponymes) ou sur le 'zrig' langagier (les phénomènes de mélanges linguistiques).

D'un autre côté, il me semble que les études linguistiques n'ont pas suscité dans le pays autant d'intérêt qu'elles l'auraient dû, surtout si l'on fait abstraction de ce qui relève spécifiquement des questions de politique linguistique.

Je ne saurais mettre un point final à cet article, cependant, sans évoquer la mémoire du très regretté Ousmane Moussa Diagana (1951-2001). Outre ses productions comme poète, il a consacré à sa langue maternelle, le soninké, des études scientifiques qui font date : un recueil sur les chants traditionnels (1990), une grammaire (1995) et un dictionnaire (2011). Nul doute que, s'il n'avait pas disparu prématurément, il aurait continué à donner le meilleur exemple aux jeunes générations ${ }^{31}$.

\footnotetext{
${ }^{30}$ Un phénomène du même ordre relie les deux toponymes Tegdaoust et Aoudaghost (cf. Taine-Cheikh $2002: 452$ et Ould Cheikh, à paraître).

${ }^{31}$ Depuis la rédaction de cet article (été 2014), j'ai appris une autre disparition qui m'a profondément attristée, celle de Mohameden Ould Ahmedou Yahya qui a été mon informateur depuis l'été 1997. C'est grâce à lui que j'ai pu faire avancer les études sur le zénaga et je veux ici témoigner de toute ma reconnaissance et de ma profonde admiration pour cet homme aux multiples qualités, tant scientifiques qu'humaines.
} 


\section{Bibliographie}

Aikhenvald, Alexandra (1988). A Structural and Typological Classification of berber Languages. Progressive Tradition in African and Oriental Studies 36-43. Berlin: Akademie Verlag.

Al-Amīn Aš-Šinqīịi, [Sid] Aḥmad (1911 (4 éd. 1989)). Al wasīṭ (pour Al wasīt fì tarāžimi 'udabā'i šinqīt). Le Caire.

Attayoub, Abdoulmobamine Khamed (2001). La tətsarrét des Ayttawari Seslem : Identification socio-linguistique d'un parler berbère non-documenté chez les touaregs de l'Azawagh (Niger). Maîtrise de berbère, Paris: INALCO.

Behnstedt, Peter \& Woidich, Manfred (2011). Wortatlas der arabischen Dialekte (WAD). Band 1. Mensch, Natur, Faune, Flora. Leiden: Brill.

Behnstedt, Peter \& Woidich, Manfred (2012). Wortatlas der arabischen Dialekte (WAD). Band II: Materielle Kultur. Leiden: Brill.

Blench, Roger (2007). Endangered Languages in West Africa. In Matthias Brenzinger (ed.), Language Diversity Endangered 140-162. Berlin - New York: Mouton de Gruyter.

Chaker, Salem (2012). Berbère et Afro-Asiatique: réflexions du berbérisant. Folia Orientalia 49, 101-120.

Cohen, David (1963). Le dialecte arabe hassānīya de Mauritanie. Paris: Klincksieck.

Cohen, David (2008). L'homme. Ses bizarres idées de bonheur... Clamecy: Cerf.

Colin, Georges S. (1930). Notes de dialectologie arabe. I. Les trois interdentales de l'arabe hispanique. II. Sur l'arabe marocain de l'époque almohade. Hesperis XI, 91-120.

Collins, Ridwan (1981-1982). Un microcosme berbère. Système verbal et satellites dans trois parlers tunisiens (1) et (2). Institut des belles-lettres arabes 148, 287-303 ; 149, 113129.

Diagana, Ousmane Moussa (1990). Chants traditionnels du pays soninké. Paris: L'Harmattan.

Diagana, Ousmane Moussa (1995). La langue soninkée : morphosyntaxe et sens. Paris: L'Harmattan.

Diagana, Ousmane Moussa (2011). Dictionnaire soninké-français (Mauritanie). Paris: Karthala.

Duchemin, Georges-Jacques \& Leriche, Albert (1952). Avant-propos. Précis sur la Mauritanie de Mokhtar Ould Hamidoun. St-Louis: IFAN-Mauritanie.

Hachimi, Atiqa (2011). Réinterprétation sociale d'un vieux parler citadin maghrébin à Casablanca. Language \& Société 138 ("Villes du Monde Arabe : variation des pratiques et des représentations"), 20-42.

Heath, Jeffrey (2002). Jewish and Muslim Dialects of Moroccan Arabic. London / New York: Routledge Curzon.

Kallas, Elie (1999). Qui est arabophone ? Gorizia: Istituto di Sociologia Internazionale di Gorizia.

Kossmann, Maarten (1999). Essai sur la phonologie du proto-berbère. Köln: Köppe.

Lux, Cécile (2011). Étude descriptive et comparative d'une langue menacée : le tetserret, langue berbère du Niger. Thèse de doctorat, Lyon: Université Lumière Lyon II.

Lux, Cécile (2013). La tetserret, langue berbère du Niger. Description phonétique, phonologique et morphologique, dans une perspective comparative. Köln: Köppe.

Moseley, Christopher (ed.), (2010), Atlas of the World's Languages in Danger, $3^{\text {rd }}$ ed., Paris: UNESCO Publishing. Online version:

$\mathrm{http}: / / \mathrm{www}$. unesco.org/culture/en/endangeredlanguages/atlas/

Naït-Zerrad, Kamal (2002). Dictionnaire des racines berbères (formes attestées), III. D-GৎY. Paris-Louvain: Peeters.

Ould Cheikh, Abdel Wedoud (1999). Vous avez dit "Histoire" ? Histoire de la Mauritanie. Essais et synthèses 7-50. Nouakchott: Université de Nouakchott \& Université de Provence. 
Ould Cheikh, Abdel Wedoud (à paraître), Aoudaghost. The Encyclopaedia of Islam, Leiden: Brill, $3^{\mathrm{e}}$ édition en cours.

Ould Taya, Moawiya (2012). Nažāt al- 'arab. Paris: al-Burāq.

Robin, Christian (2000). H53. Himyar/Himyarites. Encyclopédie berbère. Fasc. XXIII. Hiempsal - Icosium 3471-74. Aix-en-Provence: EDISUD.

Souag, Lameen (2010). The western Berber stratum in Kwarandzyey (Tabelbala, Algeria). In H. Stroomer, M. Kossmann, D. Ibriszimow \& R. Vossen (eds), Études berbères V. Essais sur des variations dialectales et autres articles. 177-189. Köln: Köppe.

Taine-Cheikh, Catherine (1983). Le passif en hassaniyya. Matériaux arabes et sudarabiques (G.E.L.L.A.S.) 1, 61-104.

Taine-Cheikh, Catherine (1988-1998). Dictionnaire Hassāniyya - Français. Paris: Geuthner.

Taine-Cheikh, Catherine (1991). L'arabe des Bị̣̂̂n, un dialecte bédouin du Maghreb occidental. In Alan S. Kaye (ed.), Semitic Studies (In honor of Wolf Leslau. On the occasion of his eighty-fifth birthday November 14th, 1991) 1528-48. Wiesbaden: Harrassowitz.

Taine-Cheikh, Catherine (1997). Les hassanophones du Maroc. Entre affirmation de soi et autoreniement. Peuples méditerranéens ("Langues et stigmatisations sociales au Maghreb") $\mathrm{n}^{\circ} 79$ (d'avril-juin 1997 [paru en 1999]), 85-102.

Taine-Cheikh, Catherine (1998). Langues, savoirs et pouvoirs en milieu maure. Nomadic Peoples ("Savoirs et pouvoirs au Sahara") n 2 (Nouvelle Série) Issues 1-2, 215-234.

Taine-Cheikh, Catherine (1999). Le zénaga de Mauritanie à la lumière du berbère commun. In M. Lamberti \& L. Tonelli (eds), Afroasiatica Tergestina 299-324. Padova, Italy: Unipress.

Taine-Cheikh, Catherine (2002). Morphologie et morphogenèse du diminutif en zénaga (berbère de Mauritanie). In K. Naït-Zarrad (ed.), Articles de linguistique berbère. Mémorial Werner Vycichl 427-454. Paris: L'Harmattan.

Taine-Cheikh, Catherine (2003a). Les valeurs du préfixe $s$ - en hassaniyya et les conditions de sa grammaticalisation. In I. Ferrando \& J. J. Sánchez Sandoval (eds), AIDA 5th Conference Proceedings, Cádiz september 2002, 103-118. Cádiz: Servicio de Publicationes Universidad de Cádiz.

Taine-Cheikh, Catherine (2003b). La corrélation de gémination consonantique en zénaga (berbère de Mauritanie). Comptes rendus du GLECS 34 (1998-2002) 2001-2003, 5-66.

Taine-Cheikh, Catherine (2005). Les numéraux en berbère. Le cas du zénaga. Studi Maghrebini (N. S.) 3 ("Studi Berberi e Mediterranei. Miscellanea offerta in onore di Luigi Serra", a curia di A. M. Di Tolli), 269-280.

Taine-Cheikh, Catherine (2007). Périphérie géographique et perméabilité aux contacts. Le cas du Maghreb. Romano-Arabica 6-7 (2006-2007) ("Peripheral Arabic Dialects", édité par G. Grigore), 159-178.

Taine-Cheikh, Catherine (2008a). Dictionnaire zénaga - français. Le berbère de Mauritanie par racines dans une perspective comparative. Köln: Köppe.

Taine-Cheikh, Catherine (2008b). Arabe(s) et berbère en contact : le cas mauritanien. In M. Lafkioui \& V. Brugnatelli (eds), Berber in Contact. Linguistic and Sociolinguistic Perspectives 113-138. Köln: Köppe.

Taine-Cheikh, Catherine (2010). Aux origines de la culture matérielle des nomades de Mauritanie. Réflexions à partir des lexiques arabes et berbères. The Maghreb Review 35, 64-88.

Taine-Cheikh, Catherine (2012). Arabe(s) et berbère en Mauritanie. Bilinguisme, diglossie et mixité linguistique. In G. Mejdell \& L. E. Edzard (eds), High vs. Low and Mixed Varieties. Status, Norms and Functions across Time and Languages 88-108. Wiesbaden: Harrassowitz.

Taine-Cheikh, Catherine (2013). Des ethnies chimériques aux langues fantômes : L'exemple des Imraguen et Nemâdi de Mauritanie. In Carole de Féral (ed.), In and out of Africa: 
Languages in Question. In Honour of Robert Nicolaï. Vol. 1. Language Contact and Epistemological Issues 137-164. Louvain-la-Neuve: Peeters.

Taine-Cheikh, Catherine (à paraître). Les voies lactées. Le lait dans l'alimentation des nomades de Mauritanie. Awal 42 ("Autour des pratiques alimentaires chez les Berbères", numéro spécial sous la dir. de M.-L. Gélard), 27-50.

Walentowitz, Saskia \& Attayoub, Abdoulmobamine Khamed (2001). La tetserrét des Touaregs Ayttawari Seslem : Un parler proche du berbère 'septentrional' chez les Touaregs de l'Azawagh (Niger). Annuaire de l'Afrique du Nord 39, 27-47. 